\title{
Histological and ultrastructural investigation of retinal microaneurysm development in diabetic patients
}

\author{
A W Stitt, T A Gardiner, D B Archer
}

\begin{abstract}
Background-Although microaneurysms are a clinicopathological hallmark of diabetic retinopathy, there have been few ultrastructural studies of these important lesions. As a result, knowledge of the mechanisms involved in the pathogenesis of microaneurysms remains fragmentary. This study provides histological and ultrastructural evidence of various stages in microaneurysm formation within the retinal vasculature.
\end{abstract}

Methods-The eyes of three type II diabetic patients, obtained within 24 hours of death, were studied by the trypsin digest technique. Eyes from two further type II diabetics were fixed in $2.5 \%$ glutaraldehyde within 12 hours of death and processed for electron microscopy.

Results-In the trypsin digest preparations, small saccular and fusiform microaneurysms were observed in the peripheral retinal. In the central retina, the microaneurysms ranged in morphology from thin walled, cellular forms to dense, acellular, hyalinised forms. Ultrastructurally, four distinct groups of microaneurysm were observed. Type I showed an extensive accumulation of

Dr A W Stitt.

Accepted for publication 9 December 1994 polymorphonuclear cells into the lumen. The endothelium remained intact, although pericytes were invariably absent. Type II microaneurysms were typified by large numbers of red blood cells (RBCs) in the lumen. Endothelial cells and pericytes were completely absent. The type III microaneurysm was also non-perfused and contained aggregates of irregularly shaped RBC profiles and RBC breakdown products. Recanalisation by new vessels into the occluded lumen was observed in one microaneurysm. Type IV microaneurysms were almost or completely sclerosed, with extensive fibrosis and lipid infiltration into the lumen and basement membrane wall.

Conclusion-This investigation describes several distinctive stages in the formation of microaneurysms during diabetic retinopathy. With reference to the pathogenesis of retinal microaneurysms, the interaction of various cell types is discussed and the significance of vascular cell death and localised hypertensive events highlighted.

(Br F Ophthalmol 1995; 79: 362-367)

The capillary microaneurysm is a clinicopathological hallmark of retinal microvascular disease in diabetic patients. ${ }^{1-3}$ These abnormalities in the retinal vasculature have been extensively studied ophthalmoscopically, where they may appear as dark red or white spots in the fundus while fluorescein angiography typically outlines perfused microaneurysms as discrete hyperfluorescent spots. ${ }^{4}$ The histological techniques of retinal flat mounting and trypsin digestion have been valuable in identifying several different microaneurysm types some of which are not visualised in the fluorescein angiogram. 1356

Despite their frequency during diabetic retinopathy, there have been relatively few histological or ultrastructural studies of microaneurysm formation. Some ultrastructural descriptions do exist; however, these early studies were limited by poor fixation and the difficulty in obtaining large quantities of high quality ultrathin sections of diabetic retinas. ${ }^{26-8}$ As a result, our knowledge of the microaneurysm
Figure 1 Trypsin digest of the retinal vasculature showing an 'early' microaneurysm (M), many of which were found in the peripheral retina. Elongated endothelial cell nucle are present within the microaneurysm $(E)$. There are many acellular capillaries $(A C)$ and pericyte ghosts (arrows) adjacent to the microaneurysm. A, retinal artery.

Magnification $\times 130$. 


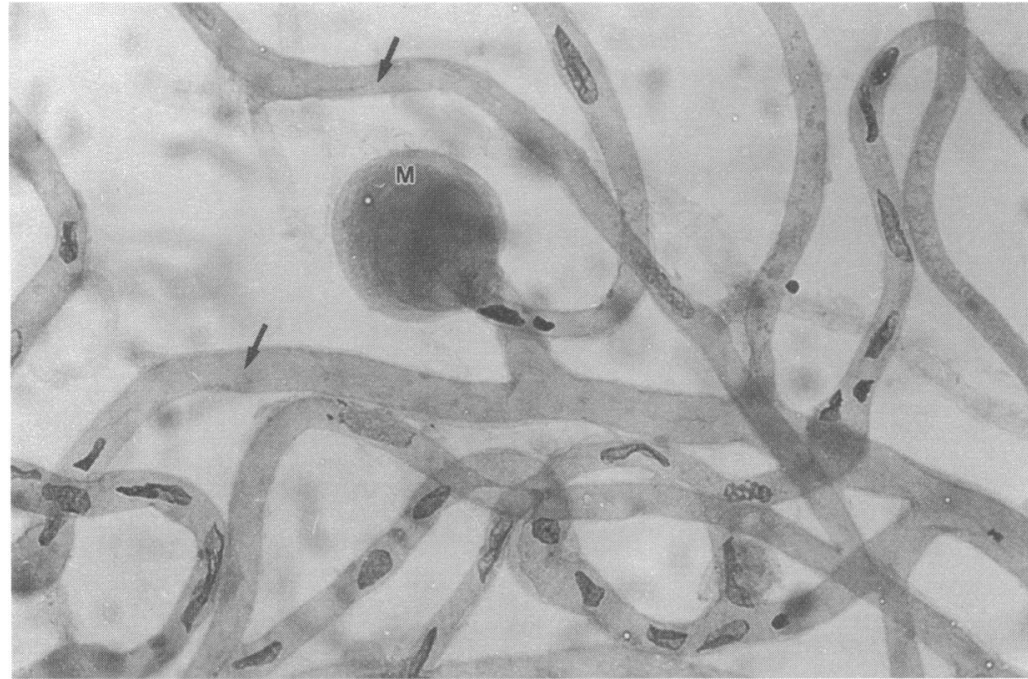

Figure 2 Trypsin digest preparation showing a completely sclerosed, periodic acid Schiff positive microaneurysm (M) from the central retina. In the immediate vicinity there are numerous acellular capillaries (small arrows). Magnification $\times 260$.

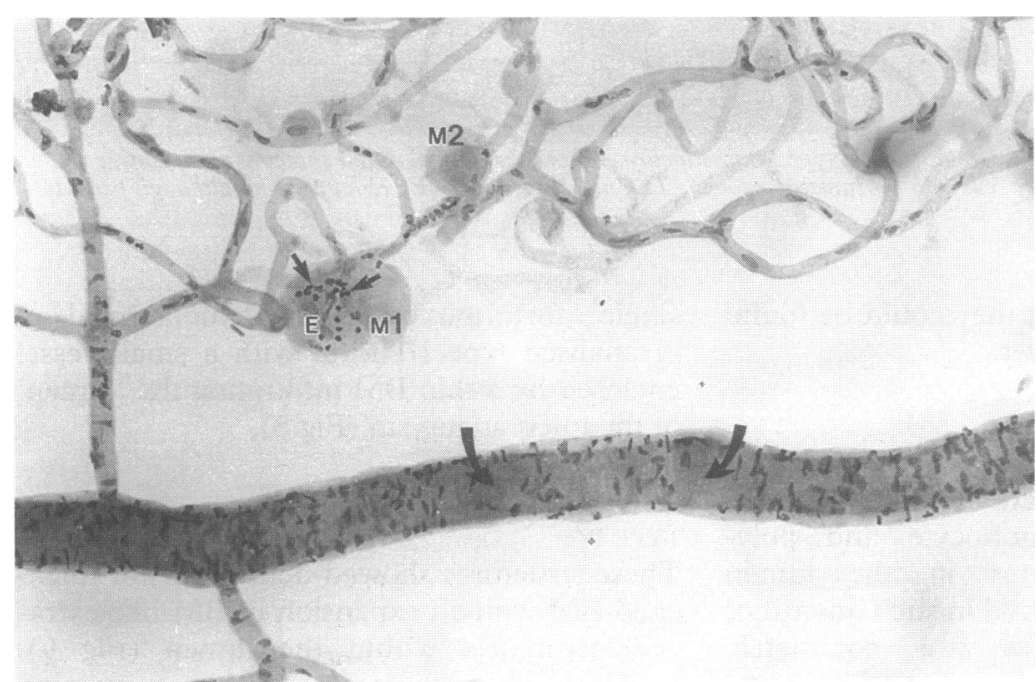

Figure 3 Trypsin digest showing microaneurysms within the central retina. One microaneurysm (M1) contains endothelial nuclei (E) and dense nuclei representative of inflammatory cells (small arrows). Another microaneurysm (M2) is acellular and sclerosed. An adjacent arteriole demonstrates distinct regions of smooth muscle cell loss (large arrows). Magnification $\times 180$.

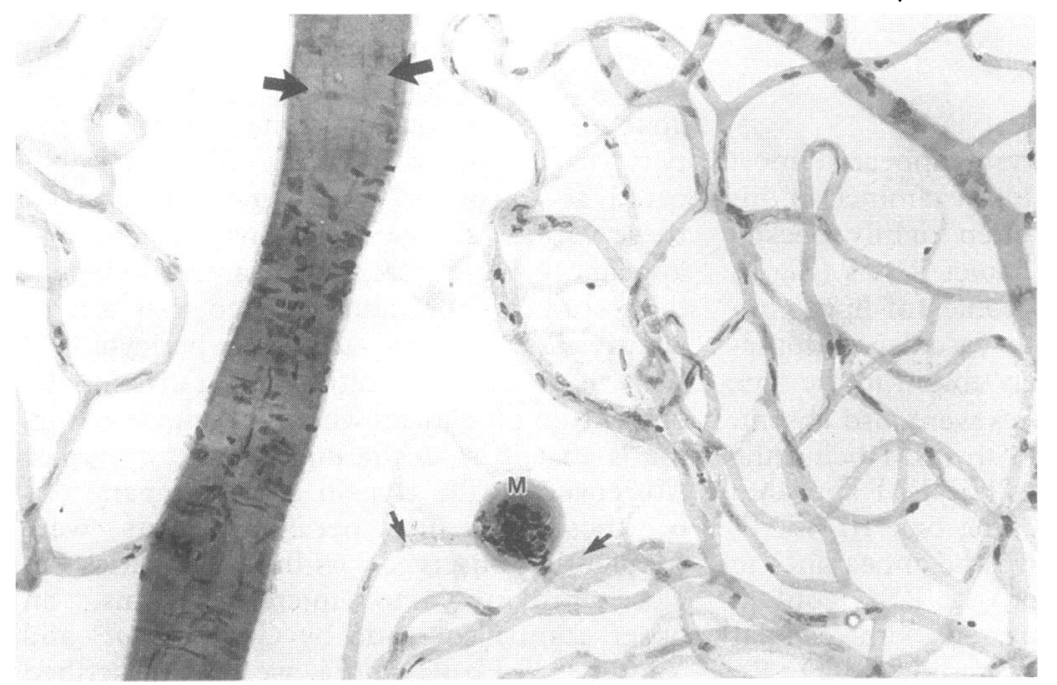

Figure 4 Trypsin digest showing a microaneurysm (M) from the central retina which contains darkly staining aggregates of red blood cells. There are many acellular capillaries in proximity to the microaneurysm (small arrows) and an adjacent arteriole shows extensive areas of smooth muscle cell loss (large arrows). Magnification $\times 130$. remains fragmentary and the mechanisms involved in their pathogenesis are largely unresolved.

In this report we provide ultrastructural insight into several distinct stages in the formation of retinal microaneurysms, which may go some way towards explaining the pathogenesis of this lesion.

\section{Materials and methods}

The eyes of three type II diabetics with pre-proliferative retinopathy were obtained within 24 hours and fixed overnight in $10 \%$ formalin in $0.1 \mathrm{M}$ phosphate buffer. The neural retina was dissected off and processed for trypsin digestion according to the method of Kuwabara and Cogan. ${ }^{9}$ After mounting, the trypsin digests were stained with periodic acid Schiff and haematoxylin.

Eyes from two further type II diabetics were obtained within 12 hours of death, both patients having received panretinal photocoagulation treatment for proliferative diabetic retinopathy. These eyes were prepared for electron microscopy by removing the anterior segment and vitreous and immersing the posterior eye cup in $2.5 \%$ glutaraldehyde in $0.1 \mathrm{M}$ cacodylate buffer ( $\mathrm{pH} \mathrm{7.4)}$ containing $10 \mathrm{mM}$ magnesium chloride. Randomly sampled blocks were dissected from the macula and non-photocoagulated areas of the central retina, post fixed in $2 \%$ osmium tetroxide, dehydrated in ascending concentrations of ethanol, and embedded in Spurr's resin.

\section{Results}

Microaneurysms were frequent in the trypsin digests and typically appeared in close proximity to arteries or arterioles and were more numerous in the central retina. Several distinct microaneurysm types were observed in the central retina, ranging from thin walled cellular types (Fig 1) to sclerosed (hyalinised) (Fig 2) forms, and corresponded to descriptions already given by deVenecia et $a l^{10}$ and Bresnick et al. ${ }^{5}$ Some of the microaneurysms contained large numbers of small, dense cell nuclei (Fig 3), while others displayed dense intraluminal aggregates of red blood cells (RBCs) (Fig 4). Proliferation of endothelial nuclei within the microaneurysm was never observed. The capillary beds in the central retina of each patient showed acellular capillaries and pericyte loss within surviving capillaries. The arteries and arterioles of these patients often showed distinct regions of smooth muscle cell loss (Figs 3 and 4). Pericyte death was frequently observed in capillaries giving rise to microaneurysms (Figs 1-4). For a description of smooth muscle cell death in these patients see Gardiner and Archer. ${ }^{11}$

Ultrastructural examination of microaneurysms from the central retina revealed a range of ultrastructural changes which could be conveniently grouped into four arbitrary stages. Most of these structures where observed in the inner layers of the retina 


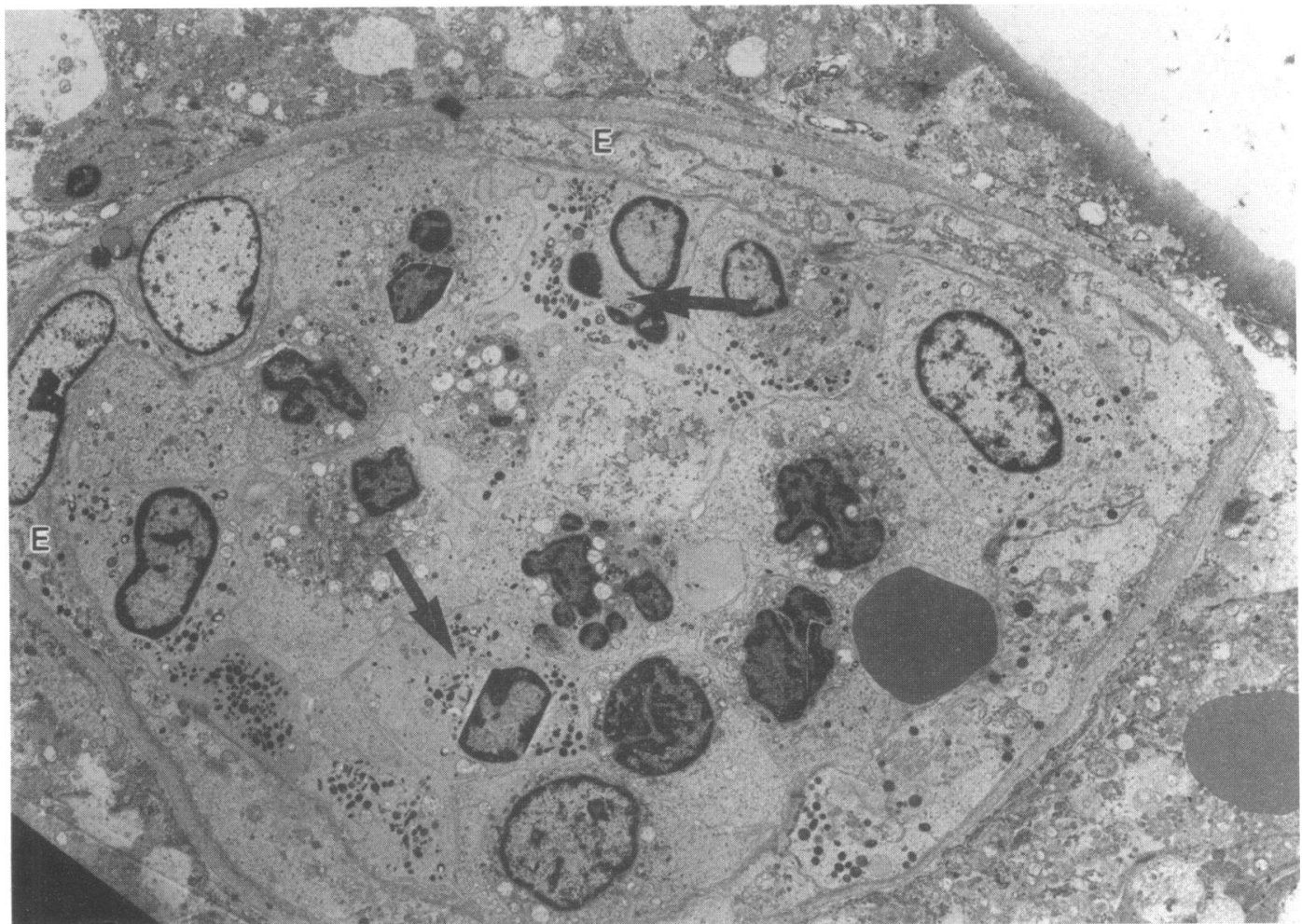

Figure 5 Transmission electron micrograph of a type I microaneurysm from the central retina showing an extensive infiltration of acute inflammatory cells into the lumen (arrows). The endothelial cells (E) remain intact although pericytes are absent. Magnification $\times 1185$.

although, less frequently, they could be found in the outer plexiform layer.

TYPE I

Sections through this microaneurysm revealed an accumulation of monocyte and polymorphonuclear leucocytes in the lumen (Fig 5). These cells occurred in such quantities that the luminal space was completely occluded. The endothelium of the vessel appeared continuous and the intercellular tight junctions remained intact. No pericyte profiles were observed at any point around the circumference of the microaneurysm. The basement membrane (BM) was slightly thickened, but no more than observed in adjacent capillary profiles.

\section{TYPE II}

This type of microaneurysm appeared most frequently in the EM sections examined in this study. The lumen was often tightly packed with dense aggregates of intact RBCs (Fig 6) and less frequently, the profiles of lipid containing macrophages. The vascular endothelium was always completely absent in the type II microaneurysm. Pericytes were also absent, with cellular debris within the BM being the only legacy of these cells. The BM appeared laminated and thickened. The type III microaneurysm was similar in size and appearance to the type II form; however, the RBC content was sparser and interspersed with cellular debris and RBC breakdown products (Fig 7). Endothelial cells, pericytes, and inflammatory cells were almost completely absent although macrophages were occasionally observed. A single fortuitous profile demonstrated a recanalised type III form with a small vessel enclosed by a thin BM infiltrating the 'lumen' of the microaneurysm (Fig 8).

TYPE IV

These structures showed dense walls of thickened BM with an expansion of BM-like extracellular matrix within the lumen (Fig 9). Profiles of degenerate lipid containing macrophages were also present.

\section{Discussion}

The retinal microaneurysm is a hallmark of diabetic retinopathy, although the pathogenesis of this vascular abnormality remains incompletely elucidated. Vascular BM damage, ${ }^{12}$ endothelial proliferation, ${ }^{13}$ changes in intraluminal rheology, ${ }^{14} 15$ and pericyte cell death ${ }^{16} 17$ have all been implicated as causal or contributory factors. Of these various changes, selective pericyte loss appears to be critical, since several independent studies have demonstrated that microaneurysms invariably lack viable pericytes. ${ }^{7} 18$ This is confirmed ultrastructurally in the current investigation, where a pericyte covering is absent in all the microaneurysm types. Moreover, in the trypsin digest preparations of these patients, pericyte ghosts were frequently observed within capillaries in close proximity to microaneurysms, an observation also made by Cogan et $a l^{3}$ and Yanoff. ${ }^{19} 20$ Furthermore, we have described pericyte cell death in capillaries giving rise to microaneurysms.

It is unlikely that pericyte cell death alone 


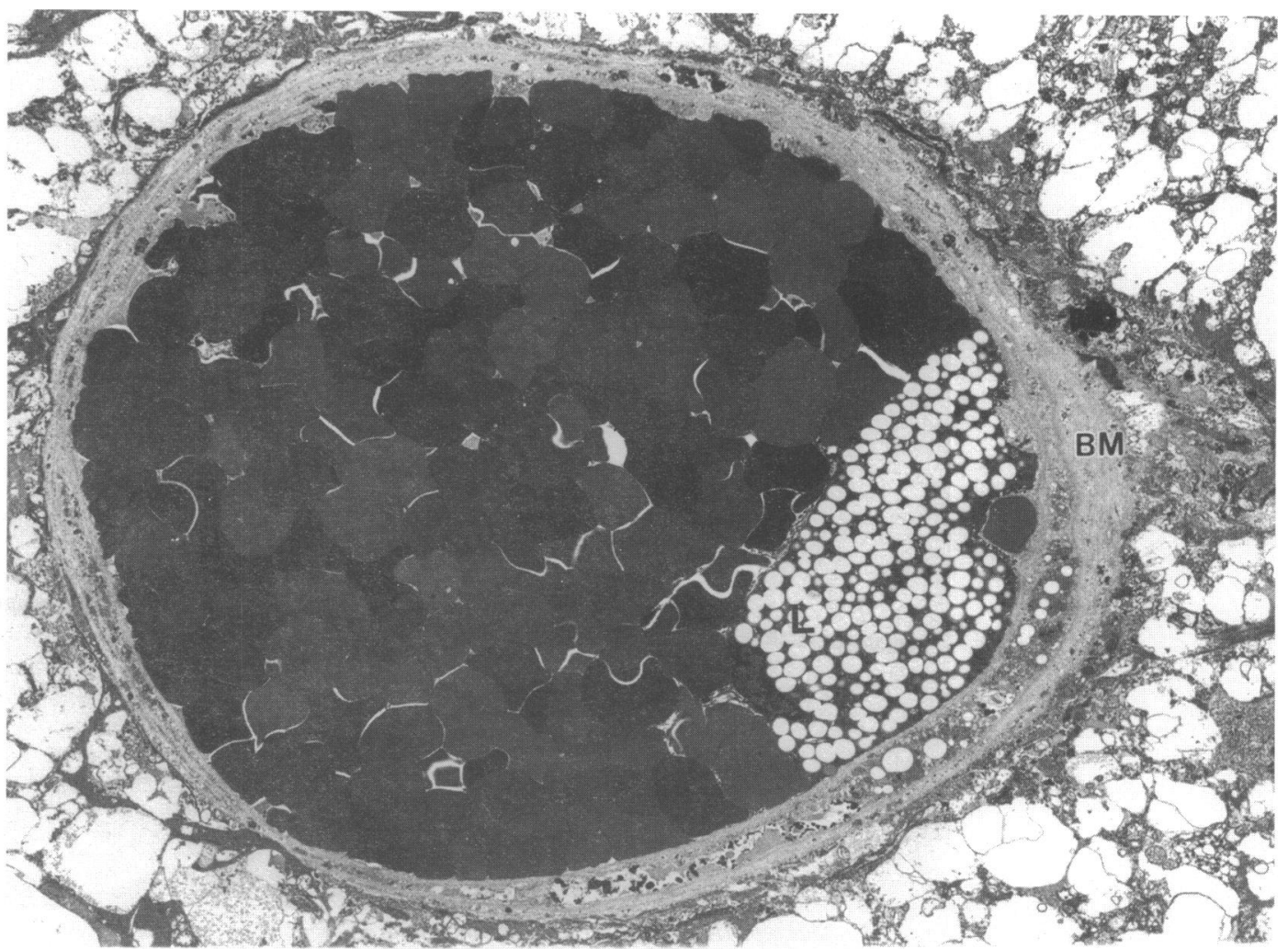

Figure 6 Transmission electron micrograph showing a type II microaneurysm which is engorged by red blood cells. The endothelial cells and pericytes are missing, while the basement membrane (BM) is somewhat thickened. This microaneurysm contains lipid deposits (L). Magnification $\times 790$.

would cause microaneurysm formation, since diabetic retinal capillaries can remain perfused and viable without a pericyte covering. Changes in retinal haemodynamics are a feature of diabetes ${ }^{21}$ and localised increases in capillary hydrostatic pressure, may account for capillary wall stretching at weak points and subsequent microaneurysm formation. ${ }^{22}$
Smooth muscle cell death has previously been described in diabetic dogs, ${ }^{23}$ and significantly, there is also selective loss of smooth muscle cells in the arteries and precapillary arterioles of the diabetic patients in the current study. ${ }^{11}$ Such changes may compromise the autoregulatory ability of the retinal vasculature and expose the downstream

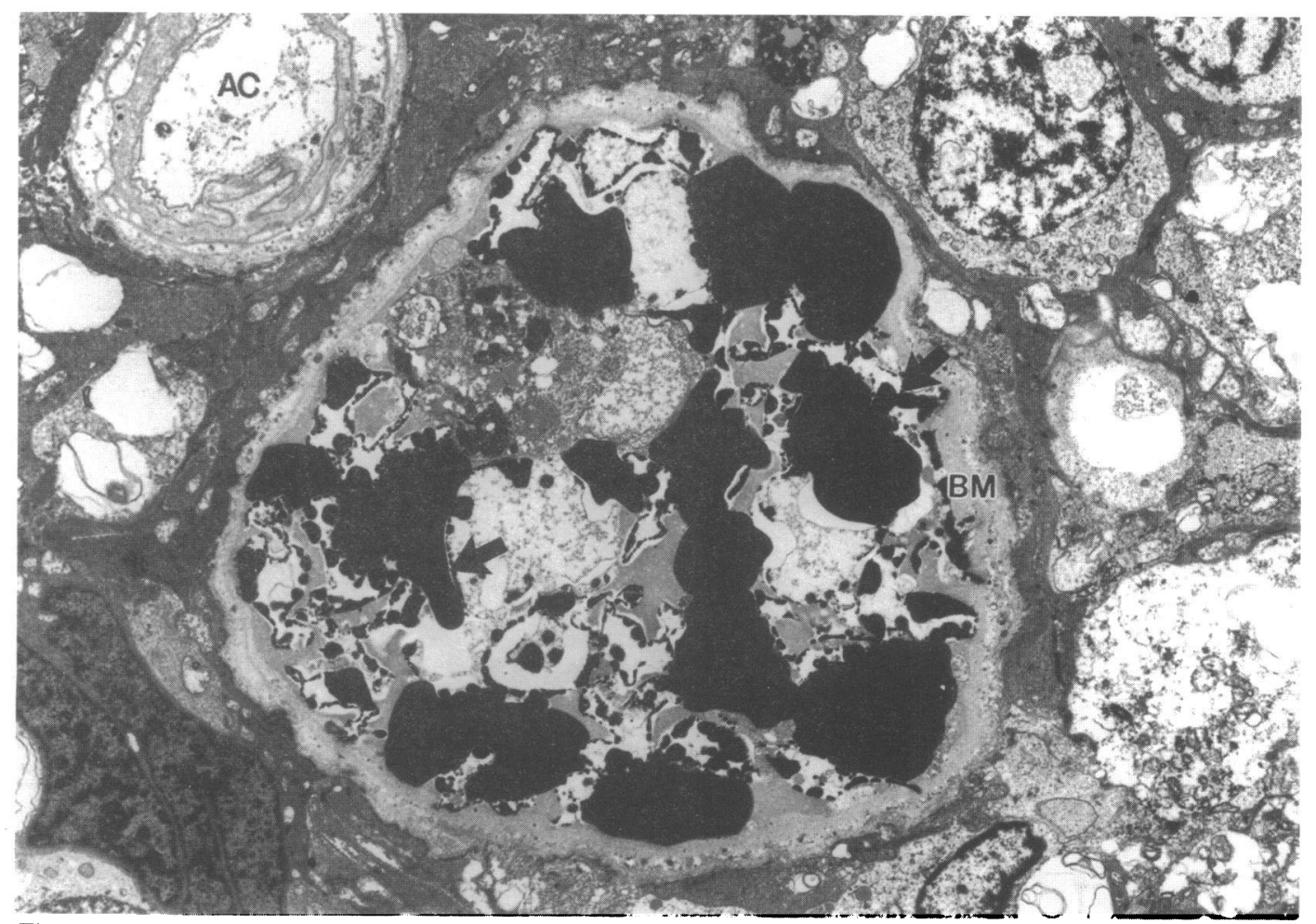

Figure 7 Transmission electron micrograph of a type III microaneurysm. This structure contains many RBCs and RBC breakdown products (arrows). The basement membrane (BM) is thickened and there is no pericyte covering. An acellular capillary profile $(A C)$ can be observed adjacent to the microaneurysm. Magnification $\times 1580$. 


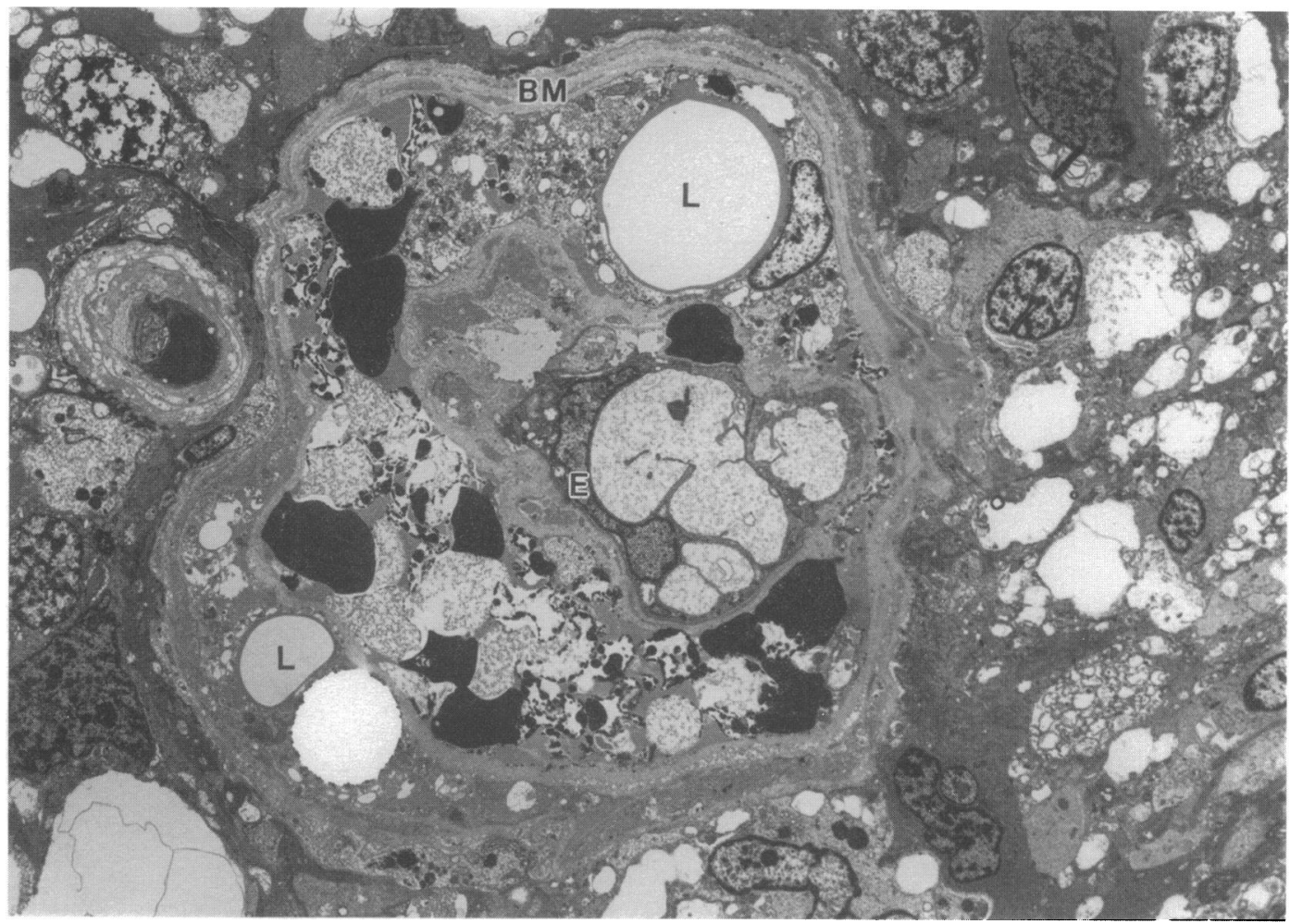

Figure 8 This type III microaneurysm appears similar to Figure 7 but contains a vessel profile within the occluded 'lumen'. This presumptive recanalising vessel has an intact endothelial lining (E) and is enclosed by basement membrane (BM). L=lipid deposits. Magnification $\times 790$.

capillary bed to uncontrolled hydrostatic pressure.

The effects of intraluminal pressure in microaneurysm formation are further underlined by the presence of retinal microaneurysms in other, non-diabetic, conditions such as hypertension, branch retinal vein occlusion, Coats' disease, sickle cell disease and certain other anaemias, albeit at much lower frequency, ${ }^{4}$ where high capillary pressure results from failure of autoregulation, obstruction of venous return, or development of high flow preferential pathways. Some of these conditions - for example, vein occlusion, would be expected to reduce oxygen availability to the retina owing to vascular

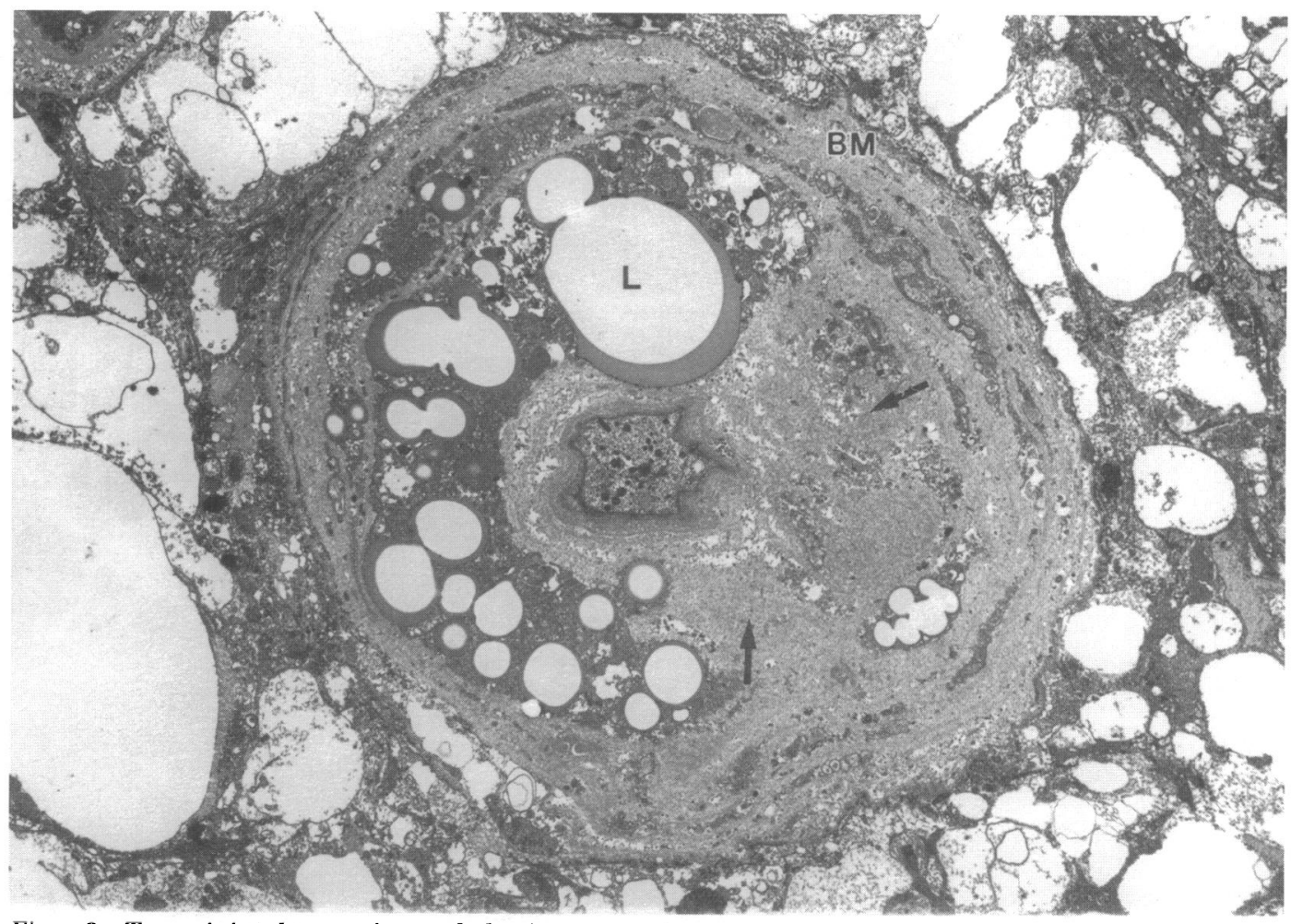

Figure 9 Transmission electron micrograph showing a type IV microaneurysm which is almost completely sclerosed and corresponds to the structures described in Figures 2 and 3. The basement membrane (BM) is very thickened and there is also fibrous infiltration (arrows) and lipid deposition $(L)$. Magnification $\times 1185$. 
stasis and/or a reduction in blood oxygen carrying capacity. This in itself may induce autoregulatory responses in the retinal arteries and thereby cause a significant increase in blood flow through the retinal capillary beds. Prolonged capillary hydrostatic pressures, coupled with impaired tissue oxygenation, could account for microaneurysm formation in the retinal vasculature at weak points in the vessel wall. In diabetes, the effect of increased retinal blood flow in association with capillary hypertension and pericyte loss probably accounts for the extensive and comprehensive formation of microaneurysms during diabetic retinopathy.

Various cell types have been described as components of microaneurysms, including newly formed endothelial cells ${ }^{13}$ and accumulated RBCs. ${ }^{7622}$ Microaneurysms have been described as focal regions of endothelial proliferation and perhaps abortive attempts at intraretinal neovascularisation. ${ }^{10}$ However, at the ultrastructural level we never found this to be the case. In the current study, the early stage, type I microaneurysm was infiltrated with large numbers of monocyte and polymorphonuclear cells. Such inflammatory cell involvement in microaneurysms has been mentioned by other workers ${ }^{16}$ but this was regarded as being of secondary importance. It is unclear what stimulus attracts such cells into these structures, although they may be responding to cytokines or adhesion molecules released from damaged endothelial cells. Indeed, it has recently been reported that the cellular adhesion molecules ICAM and VCAM are upregulated in late diabetic retinopathy. ${ }^{24} 25$ The type I microaneurysm that we describe is the only type to have an intact endothelium. It is possible that large numbers of these inflammatory cells could damage the endothelium with which they are in contact. The type II and III microaneurysms are without an endothelium, and this in itself may allow the striking accumulation of RBCs that are typical of these microvascular abnormalities. It is known that anticoagulatory factors are normally secreted by endothelial cells ${ }^{15}$ and their disappearance may be an important contributory factor in intravascular thrombus formation and the progression of microaneurysm formation.

In a clinicopathological study using fluorescein angiography and subsequent trypsin digestion, de Venecia et al ${ }^{10}$ described microaneurysms in which only a small part of the lumen was perfused. In the current investigation we describe a large microaneurysm in which a small region of the lumen had become recanalised with endothelial cells. This recanalising vessel had also secreted a BM around itself, within the lumen of the previous BM. It is likely that this reperfused structure corresponds to the microaneurysms described by de Venecia et al ${ }^{10}$ which displayed a reduced, yet significant, blood flow in the fluorescein angiogram. Microaneurysms traditionally represent a stagnated region of the retinal circulation and as a direct result would probably produce local regions of retinal hypoxia or ischaemia downstream. Growth factors are known to be released by ischaemic retina, and in localised regions of ischaemia, paracrine release of such factors may induce the recanalisation of certain microaneurysms.

1 Ashton N. Studies of the retinal capillaries in relation to diabetic and other retinopathies. Br $\mathcal{F}$ Ophthalmol 1963; 47: 521-38.

2 Bloodworth JMB. Diabetic retinopathy. Diabetes 1962; 11: $1-22$.

3 Cogan DG, Toussaint D, Kuwabara T. Retinal vascular patterns IV. Diabetic retinopathy. Arch Ophthalmol 1961;
path 66: 366-78

4 Bresnick GH, Segal P, Mattson D. Fluorescein angiopathic and clinicopathologic findings. In: Little HL, Jack RL, Patz A, Forzham PH, eds. Diabetic retinopathy. New York: Thieme-Stratton, 1983: 37-71.

5 Bresnick GH, Davis MD, Myers FL, de Venecia G. Clinicopathologic correlations in diabetic retinopathy II. Arch Ophthalmol 1977; 95: 1215-20.

6 Yamashita T, Rosen DA. Electron-microscopic study of diabetic capillary aneurysms. Arch Ophthalmol 1962; 67: diabetic

7 Bloodworth JMB, Molitor DL. Ultrastructural aspects of human and canine diabetic retinopathy. Invest Ophthalmol 1965; 4: 1037-48.

8 Toussaint D, Dustin P. Electron microscopy of normal and diabetic retinal capillaries. Arch Ophthalmol 1963; 70: 140-52.

9 Kuwabara T, Cogan DG. Studies of retinal vascular patterns: part 1. Normal architecture. Arch Ophthalmol 1960; 64: 904-11.

10 de Venecia G, Davis M, Engerman R. Clinicopathologic correlations in diabetic retinopathy I. Arch Ophthalmo 1976; 94: 1766-73.

11 Gardiner TA, Archer DB. Smooth muscle cell loss in retinal arterioles in human diabetes. Invest Ophthalmol Vis Sci 1994; 35: 1872.

12 Frank RN. On the pathogenesis of diabetic retinopathy. Ophthalmology 1984; 91: 626-34.

13 Yanoff M, Fine BS. Occular pathology: a text and atlas. New York: Harper and Row, 1975: 566-85.

14 Little HL, Sachs AH. Role of abnormal blood rheology in the pathogenesis of diabetic retinopathy. Trans Am Acad Ophthalmol Otolaryngol 1977; 83: 522-34.

15 Little HL. Alterations in blood elements in the pathogenesis of diabetic retinopathy. Ophthalmology 1981; 88: 647-54.

16 Cogan DG, Kuwabara T. The mural cell in perspective. Arch Ophthalmol 1967; 78: 133-9.

17 Speiser P, Gittelsohn AM, Patz A. Studies on diabetic retinopathy III. Influence of diabetes on intramural pericytes. Arch Ophthalmol 1968; 80: 332-8.

18 Uga S, Shimizu K, Hayashi M. Ultrastructure of retinal microaneurysms in diabetes mellitus. Acta Soc Ophthalmo fap 1977; 81: 1716-22.

19 Yanoff $M$. Diabetic retinopathy. $N$ Engl f Med 1966; 274: 1344-9.

20 Yanoff M. Ocular pathology of diabetes mellitus. $A m \mathfrak{f}$ Ophthalmol 1969; 67: 21-38.

21 Tooke JE. Microvascular haemodynamics in diabetics. Eye 1993; 7: 227-9.

22 Fryczkowski AW, Chambers RB, Craig EJ, Walker J, Davidorf FH. Scanning electron microscopic of microaneurysms in the diabetic retina. Ann Ophthalmol 1991, 23: $130-6$

23 Gardiner TA, Stitt AW, Anderson HR, Archer DB Selective loss of vascular smooth muscle cells in the retina microcirculation of diabetic dogs. $\mathrm{Br} \mathcal{F}$ Ophthalmol 1993; 78: $54-60$.

24 Tang S, Le-Ruppert KC, Gabel VP. Expression of intercellular adhesion molecule-1 (ICAM-1) and vascular cell adhesion molecule-1 (VCAM-1) on proliferating vascular endothelial cells in diabetic epiretinal membranes. endothelial cells in diabetic

25 Heidenkummer HP, Kampik A. Intercellular adhesion molecule-1 (ICAM-1) and leukocyte function-associated antigen-1 (LFA-1) expression in human epiretinal membranes. Graefes Arch Clin Exp Ophthalmol 1992; 230 483-7. 